\title{
Joint Bidding under Capacity Constraints
}

\author{
Beatriz De Otto-López \\ University of Oviedo, Oviedo, Spain \\ E-mail: bdeotto@uniovi.es \\ Received July 14, 2011; revised August 30, 2011; accepted September 7, 2011
}

\begin{abstract}
In this paper we analyze the anticompetitive effects of concentration of ownership in auction markets. We compare two different auction formats with uniform price. In the first, the price equals the highest accepted bid, whereas in the second the price equals the lowest rejected bid. For the former, and for a two-unit, two-plants, two-firms model, we find an equilibrium where all plants (all firms) bid according to a common bidding function. The concentration of the ownership has the same effect on the bidding behavior as eliminating one plant. However, the expected price is lower than the one expected in such three independent plant scenario. More surprisingly (and special to this $2 \times 2 \times 2$ case), the equilibrium is efficient. In the latter, alternative auction format, firms bids asymmetrically for its two plants. Hence, the equilibrium is inefficient. Also, with this format, we show that the market price may be arbitrarily large. Thus, and contrary to some plausible expectation base in received auction theory, a (sealed-bid) auction format in which the price for a bidder is unrelated to his bid becomes less efficient than one in which the price may coincide with that bidder's bid, when one admits that several bidders may coordinate (through ownership) their bids. The results add to a literature that favors more winner's-bid pricing rules.
\end{abstract}

Keywords: Uniform-Price Auction Formats, Capacity Constraints, Ownership Structure, Collusion

\section{Introduction}

The concentration of ownership in an industry-a smaller number of firms, each of which owns a larger size of capacity- increases market power, and this results in price increases compared to a situation in which ownership is disperse. This has been well studied and documented in decentralized markets. The purpose of this paper is to investigate whether the concentration has the same anticompetitive effect in auction markets.

This paper is motivated by some experiences in the reform of regulation of the electricity industry aiming at introducing competition at the generation level, usually characterized by a high degree of concentration. As an example of such experiences, the Spanish electricity industry is dominated by two major generators, Iberdrola and Endesa, which own most of the generation plants and set the market price at the pool near 90 per cent of the times. However, the bidding units in the pool are the generation plants, which must submit their price asks simultaneously. Obviously, the bids of the plants belonging to the same firm are not independent. In other words, firms have the ability to strategically choose the bids of their plants in a manner that can be different to the one we could expect if each plant were owned by a different firm. This ability allows the firms to increase the market price relative to a situation in which the ownership is disperse. How large is this price increase depends on the price formation rules of the mechanism.

In this paper we study a multi-unit auction mechanism for two units (the market demand), with four production plants-the bidders-with one unit production capacities, whose cost are independent draws of the same random variable. The two pants that submit the lowest bids are called to produce one unit each. We consider two different uniform-price auction formats, one with price equal to the bid of the last seller called to produce, and the other with price equal to the lowest unsuccessful bid.

We describe a concentrated ownership structure in which two firms own two plants each. Thus, a given firm decides on the bids of the two plants it owns. In principle, a bidding strategy of a firm could consist on a pair of bidding functions (one for each plant), each one depending on the costs of the two plants this firm owns. However, we show that at any symmetric equilibrium, and for either of the two auction formats, the bidding functions 
are independent in the sense that its bid depend on its cost (apart from its ranking among the plants of the same firm), and not on the cost of other plants of the same firm.

For the auction format with price equal to the highest successful bid, we show that there exists a monotone symmetric equilibrium in which the two types of pants (the more and the less efficient ones belonging to the same firm) use the same bidding function. Moreover, such function is the symmetric equilibrium bidding function of an auction for two units with the same price formation rules buy only three plants owned by independent firms. It is easy to check that this function is everywhere above the symmetric equilibrium one for the case of four independent plants. Thus, the concentration of the ownership induces the plants to bid higher compared to a situation with disperse ownership. More precisely, they bid as if there were just three plants in competition. Hence, the concentration has the same effect on the bidding behavior as eliminating one plant. As the plants bid higher with concentrated ownership, the expected price is greater than with four independent plants, but it is lower than with three independent plants. Indeed, with three plants, the price is set by the plant with the second lowest of three realizations of the cost, whereas with concentrated ownership, the price is set by the one with the second lowest of four realizations. In other words, the concentration of the ownership increases the price with respect to a situation in which the plants belong to independent firms, but not as much as eliminating one plant.

More surprisingly, this equilibrium is efficient, as all plants bid according to a monotone increasing function, and hence, by scheduling the lowest bidders, the mechanism calls to produce the plants with the lowest costs.

For the auction format with price equal to the lowest unsuccessful bid, Vickrey [1] has shown that bidding the cost is a weakly dominant strategy when bidders can at most be awarded with one unit (in our model, this is as to say that ownership is disperse). Ausubel and Cramton [2] and Engelbrecht-Wiggans and Kahn [3] analyze a mechanism in which (exchanging the roles of buyers and sellers) bidder can win more than one unit of an indivisible $\operatorname{good}^{1}$. For a bidder that desires more than one unit there is a positive probability that the bid for the second or latter units determines the price paid for the other units that he wins. Therefore, there is an incentive to bid truthfully on the first unit, but to shade the true valuation of the second and subsequent ones, in order to decrease the price of the unit it wins. Thus, there is a positive probability that the mechanism result in ex post ineffi-

\footnotetext{
${ }^{1}$ Also Brusco and Lopomo [4] analyze a multi-object version of the English oral auction with heterogeneous objects that can be sold at different prices. They find that the possibility of signaling trough the bids allows the buyers to split the objects among them at low prices.
}

cient allocations. There is also a recently increasing research analyzing alternative auction designs and pricing rules for wholesale electricity markets (see, for instance, Cramton et al. [5], Federico and Rahman [6], Fabra [7], Cramton and Stoft [8], and Tierney et al. [9]).

Our model of concentrated ownership, where the plants bid for the right to supply the market demand, is equivalent to auction models where the bidder wants more than one unit. Thus, for the auction format where the price equals the lowest rejected bid our results are in line with those in Engelbrecht-Wiggans and Kahns'; bidding the true cost of the first plant is a weakly dominant strategy, but the bid of the second plant must be above the cost. As in their model, inefficiencies arise as there is a tendency towards disseminating the units across firms more than what the relative costs would indicate. Moreover, also in line with their results, we show that there exists a continuum of monotone symmetric equilibria in which the market price is arbitrarily large.

Certainly, the efficiency result for the alternative auction format is special to the $2 \times 2 \times 2$ model we analyze (although it is also true with some other special market configurations). Nevertheless, it points to better efficiency and revenues properties of an auction format that is more similar to a "pay your bid" auction, as compared to one more similar to second price auction ${ }^{2}$. Our claims is that the auction format with price equal to the best unsuccessful bid gives larger opportunities to tacit collusion among the bidders than the format with price equal to the worse successful bid. Indeed, in the former, all equilibria are inefficient, and the bidders have the ability to coordinate on "split award" equilibria at which they can increase the price with no bound.

The rest of the paper is organized as follows. In Section 2 we describe the model and we prove the independence of the bidding functions. In Section 3 we examine the auction format with price equal to the highest successful bid, and we show that there exists an equilibrium in which all the plants bid according to the same bidding function. In Section 4 we analyze the auction with price equal to the lowest unsuccessful bid, and we show that there exists a collection of equilibria with price arbitrarily large. Section 5 contains some of the concluding remarks. The appendix contains some of the proof.

\section{The Model}

There are two firms, which own two production plants each. Each plant has production capacity for one unit.

${ }^{2}$ This is yet another example of how intuitions based on single objects auctions may be inadequate for multi-unit auctions; see for instance the discussion in Ausubel and Cramton (1998). 
The unit costs of the plants are constant. They are independent draws of a random variable with cumulative density function $F$ and distribution function $f$. The support of the distribution is $[\underline{c}, \bar{c}]$. This is common knowledge. The cost of a plant is private information to the firm that owns the plant.

The market demand of the good is equal to two units for any price.

The sellers compete in a pool mechanism for the right to supply one unit of output. Each plant submits a bid that represents the price at which this plant offers this unit. The pool ranks the bids in ascending order and calls the two plants which submit the two lowest bids to produce. The auction is a uniform price one. That means that the two plants in the schedule are paid the same price. However, we consider two auction formats which differ in the manner this uniform price is determined. In one case, the price is equal to the bid of the last plant called to produce, that is, the highest successful bid. In the second case, the market price is equal to the lowest unsuccessful bid.

\section{The Bidding Strategies}

Each firm observes the costs of the plants it owns, that we denote by $c_{1}$ and $c_{2}$, with $c_{1} \leq c_{2}$. Then, the firms simultaneously submits two bids each, $b_{1}$ and $b_{2}$, with $b_{1} \leq b_{2}$. A bidding strategy for a firm is a pair of bidding functions $b_{1}\left(c_{1}, c_{2}\right)$ and $b_{2}\left(c_{1}, c_{2}\right)$, which determines the bids of the two plants that the firm owns. It is straightforward that at any equilibrium the lowest bid $b_{1}$ must correspond to the plant with the lowest cost $c_{1}$.

We look for symmetric equilibria of these games. Our first result, which applies to both auction formats, greatly simplifies this question.

Proposition 1. In any symmetric equilibrium, the bidding functions are independent in the following sense: conditional on that the cost of a given plant is greater that (or less than) the cost of the other, its bid depends only on its cost, and not on the cost of the other plant owned by the firm. This holds for the two auction formats; with price equal to the highest successful bid and with price equal to the lowest unsuccessful bid.

Proof. See the appendix.

Thus, at any symmetric equilibrium any firm must behave as follows. First, it must observe the costs of its two plants in order to identify the one with the low cost $c_{1}$. Then, the firm assigns a bidding function to each plant, so that the plant with low cost bids according to the function $b_{1}$ (depending only on the cost of the first plant), and the plant with the high cost bids according to the function $b_{2}$ (depending only on its cost), where $b_{1}(c) \leq b_{1}(c)$ for any $c \in[\underline{c}, \bar{c}]$.
The intuition behind this result is the following. Let us consider first a mechanism in which the uniform price is equal to the bid of the last plant called to produce. The bid $b_{1}$ can affect the profits of the firm as much as it affects whether the first plant producer or not or if it affects the price this plant obtains. In particular, it cannot affect whether or not plant 2 produces or the price in the market when it happens. Hence, the bid $b_{1}$ must depend solely on $c_{1}$.

Suppose now that the firm decreases $b_{2}$ by $\varepsilon$ (with $b_{2}-\varepsilon$ still above $b_{1}$ ). The profits of the firm change only if plant 2 is at the second position before and after lowering $b_{2}$ (then the profits are reduced by $2 \varepsilon$, that is, the reduction of the price times the number of units the firm produces), or if plant 2 moves from the third to the second position, in which case the profits increase by $b_{2}-c_{2}$. As before, the changes in the profit caused by a change in $b_{2}$ do not depend on the cost $c_{1}$ of the other plant. Therefore, the optimum bidding function $b_{2}$ does not depend on $c_{1}$.

Let us consider now a mechanism with price equal to the highest unsuccessful bid. Again, a change in $b_{1}$ cannot affect the revenues or costs accruing from plant 2 . Suppose now that the firm reduces $b_{2}$ by $\varepsilon$. This change affects the profits only if plant 2 is at the third position before and after (then the profits are reduced by $\varepsilon$, as much as the market price), of if the change in $b_{2}$ moves plant 2 from the third to the second position, in which case plant 2 enters the production schedule and the profits increase by $b_{2}-c_{2}$. Again, these changes do not depend on $c_{1}$, and therefore, the optimum bidding function for plant 2 must depend only on $c_{2}$.

Next we describe the conditions which define any strictly monotone symmetric equilibrium strategies for each of the two different price mechanisms we have considered.

\section{When the Highest Successful Bid Sets the Price}

Let us consider first the case in which the price is equal to the bid of the last plant called to produce. Think of a firm with plants 1 and 2, whose costs are $c_{1}$ and $c_{2}$ respectively, which bids $b_{1}$ and $b_{2}$. Suppose that the rival firm bids according to some strictly increasing (and hence invertible) and differentiable functions $b_{1}$ and $b_{2}$ with $b_{1}(c) \leq b_{2}(c)$ for any $c$.

If both plant 1 and plant 2 are called to produce, the price is set by the second one and hence equal to $b_{2}$. Then, the profits of the firm are $2 b_{2}-c_{1}-c_{2}$. This occurs when the lowest rival bid is higher than $b_{1}^{-1}\left(b_{2}\right)$, and hence, with probability 


$$
\left[1-F\left[b_{1}^{-1}\left(b_{2}\right)\right]\right]^{2} \text {. }
$$

If plant 1 is the first one in the ranking and plant 2 is off the production schedule, then the price is equal to the lowest rival bid. The expected profits conditional on $b_{1}$ being the first on the ranking and $b_{2}$ being strictly above the second position are

$$
\frac{\int_{b_{1}^{-1}\left(b_{1}\right)}^{b_{1}^{-1}\left(b_{2}\right)}\left[b_{1}(z)-c_{1}\right] 2[1-F(z)] f(z) \mathrm{d} z}{\int_{b_{1}^{-1}\left(b_{1}\right)}^{b_{1}^{-1}\left(b_{2}\right)} 2[1-F(z)] f(z) \mathrm{d} z} .
$$

The denominator in this expression is the probability that the lowest rival bid is between $b_{1}$ and $b_{2}$.

Finally, if plant 1 is the second lowest bidder, that is, the one that sets the price, then the profits of the firm are $b_{1}-c_{1}$. This occurs when the lowest cost of the rival firm is less than $b_{1}^{-1}\left(b_{1}\right)$, and the highest is greater than $b_{2}^{-1}\left(b_{1}\right)$ (notice that $b_{2}^{-1}\left(b_{1}\right) \leq b_{1}^{-1}\left(b_{1}\right)$ ), and hence with probability

$$
\begin{aligned}
\int_{\underline{c}}^{b_{2}^{-1}\left(b_{1}\right)} 2\left[1-F\left[b_{2}^{-1}\left(b_{1}\right)\right]\right] f(z) \mathrm{d} z \\
+\int_{b_{2}^{-1}\left(b_{1}\right)}^{b_{1}^{-1}\left(b_{1}\right)} 2[1-F(z)] f(z) \mathrm{d} z,
\end{aligned}
$$

or equivalently,

$$
2 F\left[b_{1}^{-1}\left(b_{1}\right)\right]-F\left[b_{1}^{-1}\left(b_{1}\right)\right]^{2}-F\left[b_{2}^{-1}\left(b_{1}\right)\right]^{2} .
$$

Summarizing, the expected profits of a firm with costs $c_{1}$ and $c_{2}$ bidding $b_{1}$ and $b_{2}$ are

$$
\begin{aligned}
\Pi\left(b_{1}, b_{2}, c_{1}, c_{2}\right)= & \left(2 b_{2}-c_{1}-c_{2}\right)\left[1-F\left[b_{1}^{-1}\left(b_{2}\right)\right]\right]^{2}+\int_{b_{1}^{-1}\left(b_{1}\right)}^{b_{1}^{-1}\left(b_{2}\right)}\left[b_{1}(z)-c_{1}\right] 2[1-F(z)] f(z) \mathrm{d} z \\
& +\left(b_{1}-c_{1}\right)\left[F\left[b_{1}^{-1}\left(b_{1}\right)\right]\left[2-F\left[b_{1}^{-1}\left(b_{1}\right)\right]\right]-F\left[b_{2}^{-1}\left(b_{1}\right)\right]^{2}\right]
\end{aligned}
$$

Setting the partial derivative with respect to $b_{1}$ equal to zero for $b_{1}=b_{1}\left(c_{1}\right)$ and $b_{2}=b_{2}\left(c_{2}\right)$ we obtain

$$
F\left(c_{1}\right)\left[2-F\left(c_{1}\right)\right]-F\left[b_{2}^{-1}\left(b_{1}\left(c_{1}\right)\right)\right]^{2}-\left[b_{1}\left(c_{1}\right)-c_{1}\right] 2 F\left[b_{2}^{-1}\left(b_{1}\left(c_{1}\right)\right)\right] f\left[b_{2}^{-1}\left(b_{1}\left(c_{1}\right)\right)\right] D b_{2}^{-1}\left(b_{1}\left(c_{1}\right)\right)=0,
$$

where $D b_{2}^{-1}$ represents the derivative of the function $b_{2}^{-1}$.

The intuition behind this condition is the following. Suppose that the firm slightly increases $b_{1}$. This affects the profits of the firm only in two cases. First, if plant 1 is the second in the ranking before and after raising the bid, in which case the profits increase as much as the bid (the market price). This occurs with probability

$$
\begin{aligned}
& F\left(c_{1}\right)\left[2-F\left(c_{1}\right)\right]-F\left[b_{2}^{-1}\left(b_{1}\left(c_{1}\right)\right)\right]^{2} \\
& 2\left[1-F\left[b_{1}^{-1}\left(b_{2}\left(c_{2}\right)\right)\right]\right]^{2}-\left[b_{2}\left(c_{2}\right)-c_{2}\right] 2\left[1-F\left[b_{1}^{-1}\left(b_{2}\left(c_{2}\right)\right)\right]\right] f\left[b_{1}^{-1}\left(b_{2}\left(c_{2}\right)\right)\right] D b_{1}^{-1}\left(b_{2}\left(c_{2}\right)\right)=0 \\
& \text { respect to this variable equal to zero. That is }
\end{aligned}
$$

To understand this expression, again, suppose that the firm increases $b_{2}$ by an infinitesimal amount (say $\varepsilon$ ). Then, its profits change only in two cases. First, if plant 2 is at the second position in the ranking before and after raising the bid. In this case, the market price increases by $\varepsilon$, and hence, the profits of the firm increase by $2 \varepsilon$ (notice that in this situation the two plants of the firm are called to produce). This occurs with probability

$$
\left[1-F\left[b_{1}^{-1}\left(b_{2}\left(c_{2}\right)\right)\right]\right]^{2}
$$

Second, if the plant moves from the second to the third position by increasing $b_{2}$. Then, the profits of the firm fall by $b_{2}\left(c_{2}\right)-c_{2}$. This occurs with probability

$$
2\left[1-F\left[b_{1}^{-1}\left(b_{2}\left(c_{2}\right)\right)\right]\right] f\left[b_{1}^{-1}\left(b_{2}\left(c_{2}\right)\right)\right] D b_{1}^{-1}\left(b_{2}\left(c_{2}\right)\right)
$$

Second, if plant 1 was the second one in the ranking before raising the bid and moves to the third position (off the schedule) by increasing $b_{1}$. Then, the profits decrease by $b_{1}\left(c_{1}\right)-c_{1}$. This occurs with probability

$$
2 F\left[b_{2}^{-1}\left(b_{1}\left(c_{1}\right)\right)\right] f\left[b_{2}^{-1}\left(b_{1}\left(c_{1}\right)\right)\right] D b_{2}^{-1}\left(b_{1}\left(c_{1}\right)\right)
$$

Similarly, we obtain the F.O.C. with respect to $b_{2}$ by setting the partial derivative of the profit function with
These two effect must balance at the optimum bid $b_{2}\left(c_{2}\right)$. This is what the second F.O.C. represents.

The initial conditions that complete the differential system which defines the symmetric equilibrium bidding strategies are

$$
\begin{aligned}
& b_{1}(\bar{c})=b_{2}(\bar{c})=\bar{c} \\
& b_{1}(\underline{c})=b_{2}(\underline{c})
\end{aligned}
$$

The later condition is a usual one in asymmetric auctions when the support of the distribution of the cost is the same for the two type of bidders. Indeed, the differential system above and the boundary conditions define a problem which is very similar to that of an asymmetric auction with two type of bidders. In our case, each firm owns one plant of each type; the plant with the low cost 
for a given firm is of one type, say type 1, and the other is of type 2.

In our case, we know that $b_{1}(\underline{c})$ must be less or equal to $b_{2}(\underline{c})$, so that a plant of type 1 with cost $\underline{c}$ is called to produce with probability one. Suppose that $b_{1}(\underline{c})$ is strictly less than $b_{2}(\underline{c})$. Then, the price paid to plant 1 is less than $b_{2}(\underline{c})$ with some positive probability (the probability that the cost of the rival plant of type 1 is between $\underline{c}$ and $b_{1}^{-1}\left[b_{2}(\underline{c})\right]$. Plant 1 could, instead, bid exactly $b_{2}(\underline{c})$. By doing so the probability that the plant enters into operation remains unchanged, since the other firm never bids below $b_{2}(\underline{c})$ for its second plant, but the market price may raise at least to $b_{2}(\underline{c})$ with probability one. So this would be a profitable deviation. Hence, $b_{1}(\underline{c})$ must be no less than $b_{2}(\underline{c})$.

Let us now explain the first initial condition above. We need to show first that $b_{1}(c)$ must be no less than $\bar{c}$. Otherwise, a plant of type 1 and cost $\bar{c}$ would be at the second position with some positive probability (recall that $b_{1}(c) \leq b_{2}(c)$ for any $c$ and, in particular, $b_{1}(\bar{c}) \leq$ $b_{2}(\bar{c})$ and, hence, there could be some plant of type 2 whose bid is greater or equal to $\left.b_{1}(\bar{c})\right)$. In this case, plant 1 with cost $\bar{c}$ would make negative profits, as price would be less than the cost $\bar{c}$. If, instead, plant 1 bids exactly $\bar{c}$, it would make zero profits with probability one.

Now, we need to show that at any symmetric monotone equilibrium $b_{1}(\bar{c})$ must be equal to $b_{2}(\bar{c})$. Suppose not, that is, $b_{1}(\bar{c})<b_{2}(\bar{c})$, and think of a plant of type 2 with cost slightly below $\bar{c}$, say $\bar{c}-\varepsilon$, bidding something between $b_{1}(\bar{c})$ and $b_{2}(\bar{c})$ (by the continuity of the function $b_{2}$, there must exist a cost $\bar{c}-\varepsilon$ such that $b_{2}(\bar{c}-\varepsilon)$ is between $b_{1}(\bar{c})$ and $\left.b_{2}(\bar{c})\right)$. As the two plans of type 1 bid less than $b_{1}(\bar{c})$, the plant bidding $b_{2}(\bar{c}-\varepsilon)$ is called to produce with probability zero. If, instead, this plant submits a bid between $\bar{c}-\varepsilon$ and $b_{1}(\bar{c})$ (this is possible as long as $b_{1}(\bar{c}) \geq \bar{c}$ ), it would be the marginal plant with positive probability, making positive profits.

Finally, $b_{1}(\bar{c})$ must be equal to $\bar{c}$. Suppose that $b_{1}(\bar{c})>\bar{c}$. Then plant 1 with cost $\bar{c}$ is off the schedule with probability one (recall that $b_{1}(\bar{c})=b_{2}(\bar{c})$ ). If, instead, this plant submits a bid between $\bar{c}$ and $\bar{c}$, it would be called to produce with positive probability at some price above $\bar{c}$, and its expected profits would be positive.

Proposition 2. At any symmetric, strictly monotone equilibrium of the auction with price equal to the highest successful bid, the bidding functions $b_{1}$ and $b_{2}$ must satisfy the boundary conditions (4) and also it must hold that

$$
b_{1}\left(c_{1}\right)=\frac{\int_{c_{1}}^{\bar{c}} b_{2}^{-1}\left[b_{1}(z)\right] f(z) \mathrm{d} z}{1-F\left(c_{1}\right)} \text {. }
$$

Proof. See the appendix.

As $b_{1}$ is everywhere above $b_{2}$, it must hold that $b_{2}^{-1}\left[b_{1}(z)\right] \leq z$ for any $z \in[\underline{c}, \bar{c}]$. Thus, at any symmetric equilibrium, it must hold that

$$
\begin{aligned}
b_{1}\left(c_{1}\right) & =\frac{\int_{c_{1}}^{\bar{c}} b_{2}^{-1}\left[b_{1}(z)\right] f(z) \mathrm{d} z}{1-F\left(c_{1}\right)} \\
& \leq \frac{\int_{c_{1}}^{\bar{c}} z f(z) \mathrm{d} z}{1-F\left(c_{1}\right)}=b\left(c_{1}\right)
\end{aligned}
$$

where the function $b$ is a symmetric equilibrium bidding function in an auction for two objects (two units of demand) and three independent bidders, with price equal to the highest successful bid. The following proposition shows that there is a symmetric equilibrium with concentrated ownership in which the four plants bids according to the same bidding function and they all bid as if there were just three plants owned by independent firms.

Proposition 3. Bidding $b_{1}(c)=b_{2}(c)=b(c)$, where

$$
b(c)=\frac{\int_{c}^{\bar{c}} z f(z) \mathrm{d} z}{1-F(c)}
$$

constitutes an equilibrium when the price equals the highest successful bid. That is, with this auction format there exists an efficient equilibrium for the two firms, two units, two plants case.

Proof. See the appendix.

Notice that $b(c)$ coincides with the bidding strategy of three independent bidders competing for two units when the price is the highest successful bid.

The intuition behind this result is simple. The bid $b_{1}$ affects the profits of the firm only in case that plant 1 bidding $b_{1}$ is the marginal plant. And if so, plant 2 is off the schedule with probability one. That is, at the marginal position, the competitors of plant 1 are the two rival plants which behave as independent bidders using a common bidding strategy $b$. This is the same situation as if there were just three plants in competition for the first and second positions in the ranking.

Now consider the bid $b_{2}$. As before, $b_{2}$ affects the profits of the firm only in case that plant 2 is the marginal one. If so, plant 1 is the first in the ranking and the firm produces two units. The gains from a higher price following an increase in $b_{2}$ is now twice as much as the ones corresponding to higher $b_{1}$. However, the "competition" faced when increasing $b_{2}$ is also twice (two extramarginal rival plants, instead of one), and hence the probability of incurring in loses is also twice higher. Thus, the incentive of higher $b_{1}$ are exactly the same as 
the incentives for higher $b_{2}$.

Remark. Notice that this result hinges on the fact that the number of competing plants for the less efficient plant of a firm equals the number of plants of that firm.

A similar result holds for some other special market configurations. Suppose that there are $N$ firms that own $m$ plants each, which bid in an auction for $k$ units with unit price equal to the $k^{\text {th }}$ lowest bid. Then, it is easy to check that if $m(N-1)=k$, then there exists a symmetric equilibrium with all the plants bidding according to the highest successful bid and $m(N-1)+1$ bidders. In other words, the plants bid as if the other plants of the same firm where not real competitors. For a brief outline of the proof, consider the first order condition for the bid of the plant with the lowest cost $c_{1}$, given that all the plants bid according to the same function $b$, that is,

$$
\begin{aligned}
& {\left[b\left(c_{1}\right)-c_{1}\right] m(N-1)\left(\begin{array}{c}
m(N-1)-1 \\
k-1
\end{array}\right) F\left(c_{1}\right)^{k-1}\left[1-F\left(c_{1}\right)\right]^{m(N-1)-k} f\left(c_{1}\right)} \\
& =D b\left(c_{1}\right)\left(\begin{array}{c}
m(N-1) \\
k-1
\end{array}\right) F\left(c_{1}\right)^{k-1}\left[1-F\left(c_{1}\right)\right]^{m(N-1)-k+1}
\end{aligned}
$$

Here, the right hand side represents the gains from increasing the bid if the plant is at the marginal position before and after the change, and the left hand side is the reduction of the profits if by increasing the bid the plant moves from the marginal to the $k+1^{\text {th }}$ position. This condition above coincides with the first order condition of an auction for $k$ and $m(N-1)+1$ independent bidders and price equal to the $k^{\text {th }}$ lowest bid.

Analogously, the first order condition for the bid of the plant with cost $c_{h}$, the $h^{\text {th }}$ lowest cost of the firm, given that they all bid according to the same function $b$ is

$$
\begin{aligned}
& {\left[b\left(c_{h}\right)-c_{h}\right] m(N-1)\left(\begin{array}{c}
m(N-1)-1 \\
k-h
\end{array}\right) F\left(c_{h}\right)^{k-h}\left[1-F\left(c_{h}\right)\right]^{m(N-1)-1-k+h} f\left(c_{h}\right)} \\
& =h D b\left(c_{h}\right)\left(\begin{array}{c}
m(N-1) \\
k-h
\end{array}\right) F\left(c_{h}\right)^{k-h}\left[1-F\left(c_{h}\right)\right]^{m(N-1)-k+h}
\end{aligned}
$$

where the expression after $h D b\left(c_{h}\right)$ in the right hand side is the probability that the plant with cost $c_{h}$ bidding $b\left(c_{h}\right)$ is the $k^{\text {th }}$ lowest bidder. Notice that this implies that the plants with costs $c_{1}, c_{2}, \cdots, c_{h-1}$, that bid less than $b\left(c_{h}\right)$, are among the $k-1$ first positions in the ranking, and hence, they produce one unit each. Thus, if the $h^{\text {th }}$ plant is the $k^{\text {th }}$ bidder after and before increasing its bid, the profits of the firm change by $h$ times the price increase.

The condition above coincides with (6) when $m(N-1)=k$, that is, when the number of units to be sold is equal to the production capacity of $N-1$ firms. Notice that when there are just two firms this condition stipulates that the demand must coincide with the capacity of a single firm.

The fact that at the equilibrium defined by proposition 3 all the plants bid according to a common and monotone bidding function greatly simplifies the comparisons between the expected prices with concentrated and with disperse ownership. In addition, the fact that there is a unique and monotone function from costs to bids has a desirable consequence in terms of efficiency; by scheduling the lowest bidders, the mechanism calls to produce the plants with the lowest costs.
Corollary. The expected market price in the symmetric equilibrium defined by proposition 3 is below that of a symmetric equilibrium in the auction with just three independent bidders and above the price when all four plants are independent.

Summarizing, in an auction with uniform price equal to the highest successful bid, the concentration of the ownership affects the bidding behaviour of the plants in the same manner as eliminating one plant. In other words, a single plant that belongs to a larger firm does not consider the other plant of the same firm as a real competitor. Hence, it is true that the concentration increases the expected market price relative to a situation with disperse ownership, but not as much as eliminating all but one of the plants that are merged. With four plants bidding as they were only three, the price is set by the plant whose cost is the second lowest of four independent draws of the same random variable, whereas when there are only three plants in competition, the cost of the marginal plant is the second lowest of three realizations of that random variable. To illustrate this point, when the random process is uniform in the interval $[0,1]$, the expected price with four independent plants is 0.6 , whereas with concentrated ownership is 0.7 and with three independent 
plants is 0.75 .

\section{When the Lowest Unsuccessful Bid Sets the Price}

Consider now a price mechanism in which the market price is equal to the bid of the plant at the third position in the ranking, that is, the lowest unsuccessful bid. Consider a firm with two plants and costs $C_{1}$ and $C_{2}$ $\left(c_{1} \leq c_{2}\right)$, that bids $B_{1}$ for plant 1 and $B_{2}$ for plant 2 . Suppose that the rival firm bids according to some strictly increasing and differentiable functions $B_{1}$ (for the plant of type 1) and $B_{2}$ (for the plant of type 2), where $B_{1}$ is everywhere below $B_{2}$.

If plants 1 and 2 are called to produce, the price is equal to the lowest rival bid. Hence, the expected profits of the firm conditional on its two plants operating are

$$
\frac{\int_{B_{1}^{-1}\left(B_{2}\right)}^{\bar{c}}\left[2 B_{1}(z)-c_{1}-c_{2}\right] 2[1-F(z)] f(z) \mathrm{d} z}{\int_{B_{1}^{-1}\left(B_{2}\right)}^{\bar{c}} 2[1-F(z)] f(z) \mathrm{d} z},
$$

where the denominator is the probability of this event.

If only plant 1 is operative (at the first or second position in the ranking) and plant is at the fourth position, then the price is set by the plant with the highest cost of the rival firm, which bids according to $B_{2}$. Hence, the conditional expected profits are

$$
\frac{\int_{B_{2}^{-1}\left(B_{1}\right)}^{B_{2}^{-1}\left(B_{2}\right)}\left[B_{2}(z)-C_{1}\right] 2 F(z) f(z) d z}{\int_{B_{2}^{-1}\left(B_{1}\right)}^{B_{2}^{-1}\left(B_{2}\right)} 2 F(z) f(z) d z} .
$$

Again, the denominator is the probability of this event.

Finally, if plant 1 is called to run and plant 2 sets the price, the profits of the firm are $B_{2}-c_{1}$. This occurs whenever the lowest cost of the rival is below $B_{1}^{-1}\left(B_{2}\right)$ and the highest is above $B_{2}^{-1}\left(B_{1}\right)$, and hence with probability

$$
\begin{aligned}
\int_{\underline{c}}^{B_{2}^{-1}\left(B_{2}\right)} 2[ & {\left[1-F\left(B_{2}^{-1}\left(B_{2}\right)\right)\right] f(z) \mathrm{d} z } \\
& +\int_{B_{2}^{-1}\left(B_{2}\right)}^{B_{1}^{-1}\left(B_{2}\right)} 2[1-F(z)] f(z) \mathrm{d} z
\end{aligned}
$$

or equivalently,

$$
F\left[B_{1}^{-1}\left(B_{2}\right)\right]\left[2-F\left[B_{1}^{-1}\left(B_{2}\right)\right]\right]-F\left[B_{2}^{-1}\left(B_{2}\right)\right]^{2}
$$

Summarizing, the expected profits of a firm with costs $C_{1}$ and $C_{2}$ bidding $B_{1}$ and $B_{2}$, given that the rival firm bids according to $B_{1}$ and $B_{2}$ are

$$
\begin{aligned}
\Pi\left(B_{1}, B_{2}, c_{1}, c_{2}\right)= & \int_{B_{1}^{-1}\left(B_{2}\right)}^{\bar{c}}\left[2 B_{1}(z)-c_{1}-c_{2}\right] 2[1-F(z)] f(z) \mathrm{d} z+\int_{B_{2}^{-1}\left(B_{1}\right)}^{B_{2}^{-1}\left(B_{2}\right)}\left[B_{2}(z)-c_{1}\right] 2 F(z) f(z) \mathrm{d} z \\
& +\left[B_{2}-C_{1}\right]\left[F\left[B_{1}^{-1}\left(B_{2}\right)\right]\left[2-F\left[B_{1}^{-1}\left(B_{2}\right)\right]\right]-F\left[B_{2}^{-1}\left(B_{2}\right)\right]^{2}\right]
\end{aligned}
$$

As before, the equilibrium bidding functions must satisfy the F.O.C. of the problem. Setting the partial deriva- tive of $\Pi$ with respect to $B_{1}$ equal to zero at $B_{1}\left(c_{1}\right)$ we have

$$
-\left[B_{1}\left(c_{1}\right)-c_{1}\right] 2 F\left[B_{2}^{-1}\left[B_{1}\left(c_{1}\right)\right]\right] f\left[B_{2}^{-1}\left[B_{1}\left(c_{1}\right)\right]\right] D B_{2}^{-1}\left[B_{1}\left(c_{1}\right)\right]=0
$$

where $D B_{2}^{-1}$ represents the derivative of the function $B_{2}^{-1}$.

From the F.O.C. above it is clear that either $B_{1}\left(c_{1}\right)=c_{1}$ for any $c_{1}$ or $F\left[B_{2}^{-1}\left[B_{1}\left(c_{1}\right)\right]\right]=0$. On the one hand, $B_{1}\left(c_{1}\right)=c_{1}$ for any $C_{1}$ means that the plants of type 1 bid their true costs. This bidding function would be a dominant strategy if the four plants were independent (if each one were owned by a different firm). On the other hand, $F\left[B_{2}^{-1}\left[B_{1}\left(c_{1}\right)\right]\right]=0$ holds if, for any $C_{1}, B_{2}^{-1}\left[B_{1}\left(c_{1}\right)\right]$ does not belong to the support $[\underline{c}, \bar{c}]$, as it occurs if the plants of type 2 always bid above the maximum bid of the plants of type 1 . That is, if the bidding function $B_{2}$ is everywhere above $B_{1}(\bar{c})$.

Consider first the case that $B_{1}\left(c_{1}\right)=c_{1}$ for any $c_{1}$. Then, by the second F.O.C. (setting equal to zero the partial derivative with respect to $B_{2}$ at $B_{2}\left(C_{2}\right)$ ) and taking into account that $B_{1}$ is the identity function, we have

$$
-\left[B_{2}\left(c_{2}\right)-c_{2}\right] 2\left[1-F\left[B_{2}\left(c_{2}\right)\right]\right] f\left[B_{2}\left(c_{2}\right)\right]+F\left[B_{2}\left(c_{2}\right)\right]\left[2-F\left[B_{2}\left(c_{2}\right)\right]\right]-F\left(c_{2}\right)^{2}=0
$$

The intuition behind this condition is the following. By changing $B_{2}$ the firm may reduce its profits by $B_{2}\left(c_{2}\right)-C_{2}$ (if plant 2 was the second plant in the ranking and becomes the third one after the change) or in- crease the profits as much as the market price (the bid $B_{2}$ ) in case that plant 2 is at the third position before and after raising its bid. The optimum bidding function $B_{2}$ must balance this trade off for any cost $c_{2}$. 
Proposition 4. For the auction with concentrated ownership and price equal to the lowest unsuccessful bid, the following conditions

$$
\begin{aligned}
B_{1}\left(c_{1}\right)=c_{1} & \forall c_{1} \in[\underline{c}, \bar{c}], \\
B_{2}\left(c_{2}\right)-c_{2}= & \frac{F\left[B_{2}\left(c_{2}\right)\right]\left[2-F\left[B_{2}\left(c_{2}\right)\right]\right]-F\left(c_{2}\right)^{2}}{2\left[1-F\left[B_{2}\left(c_{2}\right)\right]\right] f\left[B_{2}\left(c_{2}\right)\right]} \\
& \forall c_{2} \in[\underline{c}, \bar{c}), \\
B_{2}(\bar{c})=\bar{c} &
\end{aligned}
$$

define a strictly monotone symmetric equilibrium at which $B_{2}\left(c_{2}\right)>c_{2}$ for any $c_{2}$ in $(\underline{c}, \bar{c})$.

Proof. The first order condition for $B_{2}$ is an immediate consequence of (8).

First, we need to prove that $B_{2}\left(c_{2}\right) \geq c_{2}$ for any $c_{2}$ in $[\underline{c}, \bar{c}]$. Think of a plant with cost $c_{2}$ bidding $B<c_{2}$. By bidding $C_{2}$ instead of $B$, the profits of the firm change only in two cases. First, if the plant was the second in the ranking and moves to the third position. If this was the case, the price before the change was below $c_{2}$ and plant of type 2 was producing one unit at some price below its cost. By increasing the bid, the plant increases the market price for the plant of type 1 (which was -and still is- operative) and, moreover, stops making losses with its plant of type 2 . And second, if the plant of type 2 was setting the price before the change. Then, by increasing the bid, the plant makes the market price increase (no matter the position of the plant after the change) and, hence, the profits accruing from the plant of type 1 increase too. Then, $B_{2}\left(c_{2}\right) \geq c_{2}$ for any $c_{2}$.

Now, we have to show that $B_{2}\left(c_{2}\right)>c_{2}$ for any $c_{2}$ in $(\underline{c}, \bar{c})$. Let $c_{2}$ be such that $B_{2}\left(c_{2}\right)=c_{2}$. Then, by condition (8), we have

$$
F\left(c_{2}\right)\left[2-F\left(c_{2}\right)\right]-F\left(c_{2}\right)^{2}=2 F\left(c_{2}\right)\left[1-F\left(c_{2}\right)\right]=0
$$

Thus, for this cost $c_{2}$, either $F\left(c_{2}\right)=0$ and $c_{2}=\underline{c}$, or $F\left(c_{2}\right)=1$ and $c_{2}=\bar{c}$.

It only remains to prove that $B_{2}(\bar{c})=\bar{c}$. Suppose not, and let $B_{2}(\bar{c})>\bar{c}$. Then, by the continuity of $B_{2}$, there is some cost at the left of $\bar{C}$ (say $\bar{c}-\varepsilon$ ) for which $B_{2}(\bar{c}-\varepsilon)>\bar{c}$. By condition (8), and taking into account that $f\left[B_{2}(\bar{c}-\varepsilon)\right]=f(\bar{c})=0$ and $F\left[B_{2}(\bar{c}-\varepsilon)\right]=F(\bar{c})=1$, we have $1-F(\bar{c}-\varepsilon)^{2}=0$, that is, $F(\bar{c}-\varepsilon)^{2}=1$ and $\bar{c}-\varepsilon=\bar{c}$, what is not possible. Hence, $B_{2}(\bar{c})$ must be exactly equal to $\bar{c}$.

Corollary. At any symmetric equilibrium defined by, (9) the market price is higher than in the unique dominant strategy equilibrium with four independent plants with probability one. Also, the equilibrium is inefficient.

The equilibrium is inefficient in the sense that there is a positive probability that the plants that are called to produce are not the ones with the lowest cost; if the plant that sets the price is of type 2 , it can occur that its cost is less than the most efficient rival plant which is scheduled. Moreover, in this case the market price is, with certainty, higher than when the four plants are independent-in which case the price is the third lowest cost, whereas with concentrated ownership, when a plant of type 2 sets the price it is because either it is the third most efficient one, bidding now above its cost, or either it is the second most efficient one, but bidding now above the three lowest costs. When a plant of type 1 sets the price, the allocation is efficient - the cost of the plant of type 2 that does produce is lower than the cost of that of type 1 that sets the price, as otherwise it would not have bid below that quantity, and the price is the same that would have prevailed with disperse ownership, as it is set by the third most efficient one which is of type 1 and, hence, bids its cost.

Summarizing, it the two plants of one firm are called to produce, the result of the auction process is the same that would have appeared with four independent plants. But is the mechanism calls to produce to one plant of each firm, then the price is, with certainty, higher than when ownership is disperse and, moreover, there is a positive probability that the allocation is inefficient.

Exchanging the roles of buyers and sellers, Vickrey (1962) showed that when a single bidder can obtain at most one unit (in our case this is as to say that the ownership is disperse), bidding the true valuation (the cost) is a weakly dominant strategy in this multi-unit auction where the price is determined by the best rejected bid. When a single bidder can obtain up to two units, Engelbrecht-Wiggans and Khan (1998) find an incentive to bid truthfully for the first unit (the first plant) but to shade the bid of the second one. The reason is that, with some positive probability, the second bid determines the price for the units he obtains. Our findings are in the same direction; the bid for the first plant coincides with its cost, but the second plant bids above its cost. Thus, if the plant that sets the price is of type 2, the price is greater than with disperse ownership. Moreover, there is a positive probability that the cost of this plant setting the price-that is, off the schedule- is less than the cost of the last plant called to run. Hence, at this equilibrium inefficient allocations arise with positive probability.

Let us go back to the first F.O.C. (7). As we have seen before, this condition holds if $B_{1}$ is the identity function or, else, if the function $B_{2}$ is bounded below by some upper bound of $B_{1}$. In fact, we will show that there is a collection of symmetric equilibria in which the plants of type 1 bid according to some bounded function $B_{1}$ and the plants of type 2 bid some upper bound $M$ of $B_{1}$, whatever the cost of the plant. 
Think of a firm with plants 1 and 2 with costs $c_{1}$ and $c_{2}$ whose rival firm bids according to a function $B_{1}$ bounded above by $B_{1}$ for its plant of type 1 , and submits a bid $M>\bar{B}_{1}$ for its plant of type 2 . Then, by bidding anything less than $M$ for plant 1 the firm makes sure that its plant will be called to run, and the market price will be $M$ or the bid this firm submits for plant 2 if it is below $M$. The firm has no incentive to submit a bid greater than $M$ for its first plant unless its cost $c_{1}$ is greater than $M$. So let us suppose that $M \geq \bar{C}$. It is clear that any bid greater or equal to $M$ for plant 2 is equally profitable for the firm. Moreover, if $M$ is large enough, the firm should bid exaclty $M$ for plant 2 . On the other hand, the firm should not bid anything between $B_{1}$ and $M$, since this would reduce the market price below $M$ without making plant 2 enter the production Schedule. By bidding less than $B_{1}$, say $\bar{B}_{1}-\varepsilon$, plant 2 is called to run with some positive probability, and in this case the market price $p$ is between $\bar{B}_{1}-\varepsilon$ and $B_{1}$. Suppose that this is the case. Then the profits corresponding to plant 2 increase by $p-c_{2}$, and the ones accruing from decrease by the price reduction $M-p$. If $M$ is large enough, bidding less than $B_{1}$ (and hence than $M$ ) for the plant of type 2 would reduce the profits of the firm.

Proposition 5. For the auction with concentrated ownership and price equal to the lowest unsuccessful bid, the following conditions

$$
\begin{array}{ll}
B_{1}\left(c_{1}\right) \leq \bar{B}_{1} & \forall c_{1} \in[\underline{c}, \bar{c}], \\
B_{2}\left(c_{2}\right)=M & \forall c_{2} \in[\underline{c}, \bar{c}], \\
M \geq \bar{c} \quad \text { and } \quad M \geq 2 \bar{B}_{1}-\underline{c}
\end{array}
$$

define a symmetric equilibrium strategy. At this equilibrium the market price is $M$.

Proof. Any function $B_{1}$ bounded above by $M \geq \bar{c}$ is equally profitable for the plants of type 1 . If plant 2 with cost $c_{2}$ bids $\bar{B}_{1}-\varepsilon$ instead of $M$, there is some positive probability that this plant is scheduled. This occurs when the lowest rival bid is some value $p$ between $\bar{B}_{1}-\varepsilon$ and $B_{1}$. In this case the profits of the firm change by $p-c_{2}-(M-p)=2 p-c_{2}-M$.

Hence, for $M$ to be more profitable than any bid less than $B_{1}$ for any cost $c_{2}$, it must hold that $M \geq 2 p-c_{2}$ $\forall c_{2} \in[\underline{c}, \bar{c}]$.

As $2 p-c_{2}$ is less than $2 \bar{B}_{1}-c_{2}$, the above condition holds for any $c_{2}$ whenever $M$ is greater or equal to $2 \bar{B}_{1}-c$.

Notice that any equilibrium of this type is equally inefficient; with probability $1 / 3$ the cost of one of the plants of type 2, which are never scheduled, is below the cost of one of the plants called to produce. This is the probability that the two plants with the lowest costs of the industry belong to the same firm. The expected effi- ciency losses are, then, 1/3 times the expected value of the difference between the third and the second lowest of four realizations of the random variable $c$. When $c$ is uniformly distributed on $[0,1]$, the expected efficiency loses are $1 / 15$.

At the most favourable equilibrium of this type, the market price $M$ is exactly $\bar{c}$. Suppose that $B_{1}\left(c_{1}\right)=0$ for any cost $c_{1}$, and $B_{2}\left(c_{2}\right)=\bar{C}$ for any $c_{2}$. A firm has not any incentive to bid more than zero for its first plant. By bidding anything less than $\bar{c}$ this plant is scheduled with probability one, and the price is $\bar{c}$ or the bid of its second plant if less than $\bar{c}$. And there are not incentives to bid less than $\bar{c}$ for the second plant, as this will only reduce the price for the first plant below $\bar{c}$ and the second plant is unable to enter into operation unless it submits a bid equal to zero.

To sum up, the concentration of the ownership is more harmful under this auction format with price equal to the lowest unsuccessful bid than with price equal to the lowest successful bid, both in terms of efficiency and price. Indeed, the price is, at any equilibrium of the type described by (10) in the former, with certainty, no less than the upper bound for the price in the later. To illustrate the different effects of the concentration on the price across the auction formats, when the random process is uniform in $[0,1]$, the price in the most favourable equilibria described by (10) is 1 , whereas the expected price when it is set equal to the lowest successful bid it is 0.7 . This means that in the first case, the expected price is a 66 per cent higher than with disperse ownership, whereas in the later the increment is of 16 per cent.

\section{Conclusions}

The concentration of the ownership in auction markets implies that a single bidder submits bids for the different units offered, and it may win more than one unit. It is already known (see, for instance, Ausubel and Cramton (1998)) that when a bidder can be awarded with more than one unit, uniform-price auctions for multiple units do not inherit the desirable efficiency and revenue properties of the auctions for a single object, except in very particular settings (as, for instance, with pure common values). The reason is that in these multi-unit auctions the bidders have an incentive to shade their true cost (or valuation), as their bid for one unit affects with positive probability the price of the other units they win.

Inefficiency is not a result of this shading per se, but rather a consequence of differential bid shading; for a bidder, the incentives to shade are different for the different units. As there is not a monotone mapping from costs (or valuations) to bids, inefficient outcomes arise with positive probability. 
The auction format with price equal to the best unsuccessful bid has been well studied by Ausubel and Cramton (1998) and Engelbrecht-Wiggans and Khan (1998), among others. In line with their results, and exchanging the roles of buyers and sellers, differential bid shading appears in our model as bidders have not an incentive to shade their first bid, since it cannot affect the price that this bidder gets. But there is a positive probability that the bid for the second unit determines the price of the first. Hence, the bidders increase this second bid in an attempt to increases the price they receive for the first unit. Indeed, in equilibrium, they can increase it with no bound.

In the auction format with price equal to the worst successful bid, we find that the incentives for bid shading are stronger when the ownership is concentrated than when each plant is an independent firm. This causes that the expected price is higher when ownership is concentrated.

More surprisingly, there are some special markets configurations for which we find bid shading, but not differential bid shading. In our $2 \times 2 \times 2$ case (two units, two firms and two plants each) there exists a symmetric equilibrium in which all the plants bid according to the same bidding function. More precisely, they bid as in a symmetric equilibrium for this auction format with three bidders that can win up to one unit. Of course, this function lies everywhere above the symmetric equilibrium bidding function for the case with disperse ownership, and this implies that the expected price is higher, but not as much as it would with three independent plants. Symmetry and monotonicity guarantee efficient outcomes.

Summarizing, the two auction formats we analyze create incentives to strategic bid shading when a single bidder can win several units, but, at least for this $2 \times 2 \times$ 2 and some other special market configurations, the auction format with price equal to the highest successful bid dominates any equilibrium of the former, alternative uniform-price auction format both in terms of price and in terms of efficiency.

\section{References}

[1] W. Vickrey, “Auctions and Bidding Games," Recent Advances in Game Theory, Princeton University Conference, 1962, pp. 15-29.

[2] L. Ausubel and P. Cramton, "Demand Reduction and Inefficiency in Multi-Unit Auctions,” Mimeo, University of Maryland, Baltimore, 1998.

[3] R. Engelbrecht-Wiggans and C. M. Khan, "Multi-Unit Auctions with Uniform Prices," Economic Theory, Vol. 12, No. 2, 1998, pp. 227-258. doi:10.1007/s001990050220

[4] S. Brusco and G. Lopomo, "Collusion via Signaling in Simultaneous Ascending Bid Auctions with Multiple Objects and Complementarities," The Review of Economic Studies, Vol. 69, No. 2, 2002, pp. 407-436. doi:10.1111/1467-937X.00211

[5] P. Cramton, A. E. Kahn, R. H. Porter and R. D. Tabors, "Uniform Pricing or Pay-as-Bid Pricing: A Dilemma for California and Beyond,” Electricity Journal, Vol. 14, No. 6, 2001, pp. 70-79.

[6] G. Federico and D. Rahman, "Bidding in an Electricity Pay-as-Bid Auction,” Journal of Regulatory Economics, Vol. 24, No. 2, 2003, pp. 175-211. doi:10.1023/A:1024738128115

[7] N. Fabra, "Tacit Collusion in Repeated Auctions: Uniform versus Discriminatory,” Journal of Industrial Economics, Vol. 51, No. 3, 2003, pp. 271-293. doi:10.1111/1467-6451.00201

[8] P. Cramton and S. Stoft, "Why We Need to Stick with Uniform-Price Auctions in Electricity Markets,” Electricity Journal, Vol. 20, No. 1, 2007, pp. 26-37. doi:10.1016/j.tej.2006.11.011

[9] S. Tierney, "Pay-as-Bid vs. Uniform Pricing: Discriminatory Auctions Promote Strategic Bidding and Market Manipulation,” Public Utilities Fortnightly, Vol. 146, No. 3, 2008, pp. 40-48. 


\section{Appendix}

\section{Proof of Proposition 1}

Consider first an auction with price equal to the highest successful bids. Think of a firm bidding $b_{1}$ and $b_{2}$ for its plants 1 and 2 with costs $c_{1}$ and $c_{2}$ respectively. Suppose that the rival firm bids according to some bidding functions $b_{1}$ and $b_{2}$ which depend both on the costs of the plants this firm owns.

When the two plants of the firm bidding $b_{1}$ and $b_{2}$ are called to run, then the market price is set by the plant bidding $b_{2}$. Hence, the expected profits of the firm conditional on that the two plants are called into operation are $2 b_{2}-c_{1}-c_{2}$. This occurs whenever the two rival plants bid above $b_{2}$. That is, when the costs of the rival, $t_{1}$ and $t_{2}$, are such that $b_{1}\left(t_{1}, t_{2}\right) \geq b_{2}$. Or equivalently, if $\left(t_{1}, t_{2}\right) \in u_{1}\left(b_{2}\right)$ where

$$
u_{1}\left(b_{2}\right)=\left\{\left(t_{1}, t_{2}\right) \in[\underline{c}, \bar{c}] \times[\underline{c}, \bar{c}] / b_{1}\left(t_{1}, t_{2}\right) \geq b_{2}\right\}
$$

This is the upper set corresponding to the value $b_{2}$ of the function $b_{1}$. In general,

$$
u_{i}(k)=\left\{\left(t_{1}, t_{2}\right) \in[\underline{c}, \bar{c}] \times[\underline{c}, \bar{c}] / b_{i}\left(t_{1}, t_{2}\right) \geq k\right\}
$$

Thus, the firm bidding $b_{1}$ and $b_{2}$ will produce two units with probability $\iint_{u_{1}\left(b_{2}\right)} f\left(z_{1}\right) f\left(z_{2}\right) \mathrm{d} z_{1} \mathrm{~d} z_{2}$, where $\iint_{u_{1}\left(b_{2}\right)}$ represents the double integral over the set $u_{1}\left(b_{2}\right)$.

If the plant with cost $c_{1}$ is the first in the ranking and the one of type 2 is at the third of fourth position, the price is set by the rival plant of type 1 , that bids according to $b_{1}$. This occurs when the lowest rival bid is below $b_{2}$ and above $b_{1}$. That is, if $\left(t_{1}, t_{2}\right) \in l_{1}\left(b_{2}\right) \cap u_{1}\left(b_{1}\right)$, where $l_{1}\left(b_{2}\right)$ is the lower set corresponding to the value $b_{2}$ of the function $b_{1}$. In general,

$$
l_{i}(k)=\left\{\left(t_{1}, t_{2}\right) \in[\underline{c}, \bar{c}] \times[\underline{c}, \bar{c}] / b_{i}\left(t_{1}, t_{2}\right) \leq k\right\}
$$

Hence, the expected profits of the firm conditional on that the price is set by the lowest rival bid are

$$
\frac{\iint_{l_{1}\left(b_{2}\right) \cap u_{1}\left(b_{1}\right)}\left[b_{1}\left(z_{1}, z_{2}\right)-c_{1}\right] f\left(z_{1}\right) f\left(z_{2}\right) \mathrm{d} z_{1} \mathrm{~d} z_{2}}{\iint_{l_{1}\left(b_{2}\right) \cap u_{1}\left(b_{1}\right)} f\left(z_{1}\right) f\left(z_{2}\right) \mathrm{d} z_{1} \mathrm{~d} z_{2}}
$$

The denominator in this expression is the probability that the lowest rival bid is between $b_{1}$ and $b_{2}$.

Finally, if the plant that bids $b_{1}$ is the one that sets the price, this is the only plant of the firm which is called to run. The profits of the firm are $b_{1}-c_{1}$ with probability

$$
\iint_{l_{1}\left(b_{1}\right) \cap u_{2}\left(b_{1}\right)} f\left(z_{1}\right) f\left(z_{2}\right) \mathrm{d} z_{1} \mathrm{~d} z_{2}
$$

This is the probability that the bid $b_{1}$ is between the two rival bids, and hence, at the second position in the ranking.

Summarizing, the expected profits of a firm with cost $c_{1}$ and $c_{2}$ bidding $b_{1}$ and $b_{2}$, given that the rival firm bids according to the functions $b_{1}$ and $b_{2}$ are

$$
\begin{aligned}
& \Pi\left(b_{1}, b_{2}, c_{1}, c_{2}\right)=\left(2 b_{2}-c_{1}-c_{2}\right) \iint_{u_{1}\left(b_{2}\right)} f\left(z_{1}\right) f\left(z_{2}\right) \mathrm{d} z_{1} \mathrm{~d} z_{2} \\
& +\iiint_{l_{1}\left(b_{2}\right) \cap u_{1}\left(b_{1}\right)}\left[b_{1}\left(z_{1}, z_{2}\right)-c_{1}\right] f\left(z_{1}\right) f\left(z_{2}\right) \mathrm{d} z_{1} \mathrm{~d} z_{2} \\
& +\left(b_{1}-c_{1}\right) \iiint_{l_{1}\left(b_{1}\right) \cap u_{2}\left(b_{1}\right)} f\left(z_{1}\right) f\left(z_{2}\right) \mathrm{d} z_{1} \mathrm{~d} z_{2}
\end{aligned}
$$

Differentiating with respect to $b_{1}$ and setting this derivative equal to zero we get the first F.O.C. of the problem, which his

$$
\begin{aligned}
& \frac{\partial \iiint_{l_{1}\left(b_{2}\right) \cap u_{1}\left(b_{1}\right)}\left[b_{1}\left(z_{1}, z_{2}\right)-c_{1}\right] f\left(z_{1}\right) f\left(z_{2}\right) \mathrm{d} z_{1} \mathrm{~d} z_{2}}{\partial b_{1}} \\
& +\iiint_{l_{1}\left(b_{1}\right) \cap u_{2}\left(b_{1}\right)} f\left(z_{1}\right) f\left(z_{2}\right) \mathrm{d} z_{1} \mathrm{~d} z_{2} \\
& +\left(b_{1}-c_{1}\right) \frac{\partial \iiint_{l_{1}\left(b_{1}\right) \cap u_{2}\left(b_{1}\right)} f\left(z_{1}\right) f\left(z_{2}\right) \mathrm{d} z_{1} \mathrm{~d} z_{2}}{\partial b_{1}}=0
\end{aligned}
$$

Clearly, this condition does not depend on $b_{2}$ neither on $c_{2}$ (notice that, although the integral in the second term of the expected profits depends on $b_{2}$, its derivative with respect to $b_{1}$ does not, as the only frontier that changes when $b_{1}$ changes is that of the set $u_{1}\left(b_{1}\right)$ ). Thus, the bidding function $b_{1}$ for the plant of type 1 depends only on the cost of this plant, and not on the cost of the second plant of the firm.

Taking this into account, and setting the derivative of the expected profits with respect to $b_{2}$ equal to zero we have the second F.O.C., which is

$$
\begin{aligned}
& 2 \iint_{u_{1}\left(b_{2}\right)} f\left(z_{1}\right) f\left(z_{2}\right) \mathrm{d} z_{1} \mathrm{~d} z_{2} \\
& +\left(2 b_{2}-c_{1}-c_{2}\right) \frac{\partial \iint_{u_{1}\left(b_{2}\right)} f\left(z_{1}\right) f\left(z_{2}\right) \mathrm{d} z_{1} \mathrm{~d} z_{2}}{\partial b_{2}} \\
& +\frac{\partial \iiint_{l_{1}\left(b_{2}\right) \cap u_{1}\left(b_{1}\right)} b_{1}\left(z_{1}\right) f\left(z_{1}\right) f\left(z_{2}\right) \mathrm{d} z_{1} \mathrm{~d} z_{2}}{\partial b_{2}}=0 \\
& -c_{1} \frac{\partial \iint_{1}\left(b_{2}\right) \cap u_{1}\left(b_{1}\right)}{f\left(z_{1}\right) f\left(z_{2}\right) \mathrm{d} z_{1} \mathrm{~d} z_{2}}
\end{aligned}
$$


As $u_{1}\left(b_{2}\right)$ and the interior of $l_{1}\left(b_{2}\right)$ are complementary sets, it holds that

$$
\frac{\partial \iint_{u_{1}\left(b_{2}\right)} f\left(z_{1}\right) f\left(z_{2}\right) \mathrm{d} z_{1} \mathrm{~d} z_{2}}{\partial b_{2}}=-\frac{\partial \iiint_{l_{1}\left(b_{2}\right) \cap u_{1}\left(b_{1}\right)} f\left(z_{1}\right) f\left(z_{2}\right) \mathrm{d} z_{1} \mathrm{~d} z_{2}}{\partial b_{2}}
$$

Also, as the frontier of the set $l_{1}\left(b_{2}\right)$ is $\left\{c \in[\underline{c}, \bar{c}] / b_{1}(c)=b_{2}\right\}$, we have that

$$
\begin{aligned}
\frac{\partial \iint_{l_{1}\left(b_{2}\right) \cap u_{1}\left(b_{1}\right)} b_{1}\left(z_{1}\right) f\left(z_{1}\right) f\left(z_{2}\right) \mathrm{d} z_{1} \mathrm{~d} z_{2}}{\partial b_{2}} & =b_{1}\left(c \in[\underline{c}, \bar{c}] / b_{1}(c)=b_{2}\right) \frac{\partial \iiint_{l_{1}\left(b_{2}\right) \cap u_{1}\left(b_{1}\right)} f\left(z_{1}\right) f\left(z_{2}\right) \mathrm{d} z_{1} \mathrm{~d} z_{2}}{\partial b_{2}} \\
& =b_{2} \frac{\partial \iiint_{l_{1}\left(b_{2}\right) \cap u_{1}\left(b_{1}\right)} f\left(z_{1}\right) f\left(z_{2}\right) \mathrm{d} z_{1} \mathrm{~d} z_{2}}{\partial b_{2}}
\end{aligned}
$$

Thus, we can rewrite the second F.O.C. as

$$
\begin{aligned}
& 2 \iint_{u_{1}\left(b_{2}\right)} f\left(z_{1}\right) f\left(z_{2}\right) \mathrm{d} z_{1} \mathrm{~d} z_{2} \\
& +\left(b_{2}-c_{2}\right) \frac{\partial \iint_{u_{1}\left(b_{2}\right)} f\left(z_{1}\right) f\left(z_{2}\right) \mathrm{d} z_{1} \mathrm{~d} z_{2}}{\partial b_{2}} \\
& =0
\end{aligned}
$$

Now, and similarly to before, this condition defines the bidding function $b_{2}$ as depending solely on $c_{2}$.

The proof for the auction format with price equal to the lowest unsuccessful bid is analogous. In this case, the expected profits of a firm with costs $C_{1}$ and $C_{2}$ bidding $B_{1}$ and $B_{2}$, given that the rival firm bid according to some functions $B_{1}$ and $B_{2}$ (which, in principle, depend on the two costs of the firm) are

$$
\begin{aligned}
& \Pi\left(B_{1}, B_{2}, c_{1}, c_{2}\right) \\
& =\iint_{U_{1}\left(B_{2}\right)}\left[2 B_{1}\left(z_{1}, z_{2}\right)-c_{1}-c_{2}\right] f\left(z_{1}\right) f\left(z_{2}\right) \mathrm{d} z_{1} \mathrm{~d} z_{2} \\
& +\iiint_{U_{2}\left(B_{1}\right) \cap L_{2}\left(B_{2}\right)}\left[B_{2}\left(z_{1}, z_{2}\right)-c_{1}\right] f\left(z_{1}\right) f\left(z_{2}\right) \mathrm{d} z_{1} \mathrm{~d} z_{2} \\
& +\left(B_{2}-C_{1}\right) \iiint_{L_{1}\left(B_{2}\right) \cap U_{2}\left(B_{2}\right)} f\left(z_{1}\right) f\left(z_{2}\right) \mathrm{d} z_{1} \mathrm{~d} z_{2}
\end{aligned}
$$

where $U$ and $L$ represent the upper and lower sets of the functions $B_{1}$ and $B_{2}$. Setting the derivative with respect to $B_{1}$ equal to zero, we have that

$$
\frac{\partial \iint_{U_{2}\left(B_{1}\right) \cap L_{2}\left(B_{2}\right)}\left[B_{2}\left(z_{1}, z_{2}\right)-C_{1}\right] f\left(z_{1}\right) f\left(z_{2}\right) \mathrm{d} z_{1} \mathrm{~d} z_{2}}{\partial B_{1}}=0
$$

Similarly to before, as the frontier of $U_{2}\left(B_{1}\right)$ is the set $\left\{\left(t_{1}, t_{2}\right) / B_{2}\left(t_{1}, t_{2}\right)=B_{1}\right\}$, this is equivalent to

$$
\left[B_{2}\left[\left(t_{1}, t_{2}\right) / B_{2}\left(t_{1}, t_{2}\right)=B_{1}\right]-c_{1}\right] \frac{\partial \iiint_{U_{2}\left(B_{1}\right) \cap L_{2}\left(B_{2}\right)} f\left(z_{1}\right) f\left(z_{2}\right) \mathrm{d} z_{1} \mathrm{~d} z_{2}}{\partial B_{1}}=\left[B_{1}-c_{1}\right] \frac{\partial \iiint_{U_{2}\left(B_{1}\right) \cap L_{2}\left(B_{2}\right)} f\left(z_{1}\right) f\left(z_{2}\right) \mathrm{d} z_{1} d \mathrm{~d}_{2}}{\partial B_{1}}=0
$$

Again, the function $B_{1}$ only depends on the cost of the plant of type 1 .
Setting now the derivative of the expected profits with respect to $B_{2}$ equal to zero, we have

$$
\begin{aligned}
& \frac{\partial \iint_{U_{1}\left(B_{2}\right)}\left[2 B_{1}\left(z_{1}, z_{2}\right)-c_{1}-c_{2}\right] f\left(z_{1}\right) f\left(z_{2}\right) \mathrm{d} z_{1} \mathrm{~d} z_{2}}{\partial B_{2}}+\frac{\partial \iiint_{U_{2}\left(B_{1}\right) \cap L_{2}\left(B_{2}\right)}\left[B_{2}\left(z_{1}, z_{2}\right)-c_{1}\right] f\left(z_{1}\right) f\left(z_{2}\right) \mathrm{d} z_{1} \mathrm{~d} z_{2}}{\partial B_{2}} \\
& +\iiint_{L_{1}\left(B_{2}\right) \cap U_{2}\left(B_{2}\right)} f\left(z_{1}\right) f\left(z_{2}\right) d z_{1} d z_{2}+\left(B_{2}-C_{1}\right) \frac{\partial \iint_{L_{1}\left(B_{2}\right) \cap U_{2}\left(B_{2}\right)} f\left(z_{1}\right) f\left(z_{2}\right) \mathrm{d} z_{1} \mathrm{~d} z_{2}}{\partial B_{2}}=0
\end{aligned}
$$

That is,

$$
\left(B_{2}-C_{2}\right) \frac{\partial \iint_{U_{1}\left(B_{2}\right)} f\left(z_{1}\right) f\left(z_{2}\right) \mathrm{d} z_{1} \mathrm{~d} z_{2}}{\partial B_{2}}+\iiint_{L_{1}\left(B_{2}\right) \cap U_{2}\left(B_{2}\right)} f\left(z_{1}\right) f\left(z_{2}\right) \mathrm{d} z_{1} \mathrm{~d} z_{2}=0
$$

and $B_{2}$ depends on $C_{2}$ and not on the cost of the plant of type 1. 


\section{Proof of Proposition 2}

Consider the F.O.C (3)

$$
2\left[1-F\left[b_{1}^{-1}\left(b_{2}\left(c_{2}\right)\right)\right]\right]^{2}-\left[b_{2}\left(c_{2}\right)-c_{2}\right] 2\left[1-F\left[b_{1}^{-1}\left(b_{2}\left(c_{2}\right)\right)\right]\right] f\left[b_{1}^{-1}\left(b_{2}\left(c_{2}\right)\right)\right] D b_{1}^{-1}\left(b_{2}\left(c_{2}\right)\right)=0
$$

As the bidding functions $b_{1}$ and $b_{2}$ cross at the upper and lower bound of the support of the distribution (see the boundary conditions of this problem), for any given cost $c$ there is a cost $c_{2}$ such that $b_{2}\left(c_{2}\right)=b_{1}(c)$. The condition above at this cost $c_{2}=b_{2}^{-1}\left[b_{1}(c)\right]$ is

$$
D b_{1}(c)[1-F(c)]-b_{1}(c) f(c)=-b_{2}^{-1}\left[b_{1}(c)\right] f(c)
$$

Integrating this expression in the interval $[c, \bar{c}]$ we have

$$
b_{1}(c)=\frac{\int_{c}^{\bar{c}} b_{2}^{-1}\left[b_{1}(z)\right] f(z) \mathrm{d} z}{1-F(c)}
$$

as we wanted to prove.

\section{Proof of Proposition 3}

Suppose that one of the firms uses the same bidding function $b$ for its two plants, and this function $b$ is defined by

$$
b(c)=\frac{\int_{c}^{\bar{c}} z f(z) \mathrm{d} z}{1-F(c)} .
$$

By setting $b_{1}(c)=b_{2}(c)=b(c)$ in (1), the expected profits of a firm with costs $c_{1}$ and $c_{2}$ bidding $b_{1}$ and $b_{2}$, we have

$$
\begin{aligned}
& \Pi\left(b_{1}, b_{2}, c_{1}, c_{2}\right)=\left(2 b_{2}-c_{1}-c_{2}\right)\left[1-F\left[b^{-1}\left(b_{2}\right)\right]\right]^{2} \\
& +\int_{b^{-1}\left(b_{1}\right)}^{b^{-1}\left(b_{2}\right)}\left[b(z)-c_{1}\right] 2[1-F(z)] f(z) d z \\
& +\left(b_{1}-c_{1}\right) 2 F\left[b^{-1}\left(b_{1}\right)\right]\left[1-F\left[b^{-1}\left(b_{1}\right)\right]\right]
\end{aligned}
$$

The first F.O.C. of this problem (setting equal to zero the partial derivative with respect to $b_{1}$ ) is

$$
\begin{aligned}
& 2 F\left[b^{-1}\left(b_{1}\right)\right]\left[1-F\left[b^{-1}\left(b_{1}\right)\right]\right] \\
& -2\left(b_{1}-c_{1}\right) F\left[b^{-1}\left(b_{1}\right)\right] f\left[b^{-1}\left(b_{1}\right)\right] D b^{-1}\left(b_{1}\right)=0
\end{aligned}
$$

Or equivalently, the optimum $b_{1}$ for cost $c_{1}$ must satisfy

$$
\left[1-F\left[b^{-1}\left(b_{1}\right)\right]\right]=\left(b_{1}-c_{1}\right) f\left[b^{-1}\left(b_{1}\right)\right] D b^{-1}\left(b_{1}\right)
$$

By the expression which defines the function $b$, we know that

$$
[1-F(c)] b(c)=\int_{c}^{\bar{c}} z f(z) \mathrm{d} z
$$

Differentiating this expression,

$$
[1-F(c)] D b(c)-b(c) f(c)=-c f(c)
$$

At $c=b^{-1}\left(b_{1}\right)$, and taking into account that $\left[D b^{-1}\left(b_{1}\right)\right]^{-1}=D b\left[b^{-1}\left(b_{1}\right)\right]$, we have

$$
1-F\left[b^{-1}\left(b_{1}\right)\right]=\left[b_{1}-b^{-1}\left(b_{1}\right)\right] f\left[b^{-1}\left(b_{1}\right)\right] D b^{-1}\left(b_{1}\right)
$$

Substituting this expression in the first F.O.C. above, it must hold that $b_{1}-b^{-1}\left(b_{1}\right)=b_{1}-c_{1}$, that is, $b_{1}=b\left(c_{1}\right)$.

Hence, the optimum bid $b_{1}$ for the cost $c_{1}$ is given by the bidding function $b$. Or, in other words, if a given firm bids according to $b$ for its two plants, then, the other firm must bid likewise for its low cost plant.

Consider now the second first order condition which we obtain by setting equal to zero the differential of the expected profits (11). That is,

$$
2\left[1-F\left[b^{-1}\left(b_{2}\right)\right]\right]^{2}-\left(b_{2}-c_{2}\right) 2\left[1-F\left[b^{-1}\left(b_{2}\right)\right]\right] f\left[b^{-1}\left(b_{2}\right)\right] D b^{-1}\left(b_{2}\right)=0
$$

or equivalently,

$$
\left[1-F\left[b^{-1}\left(b_{2}\right)\right]\right]=\left(b_{2}-c_{2}\right) f\left[b^{-1}\left(b_{2}\right)\right] D b^{-1}\left(b_{2}\right)
$$

This expression is equivalent to (12) for the bid $b_{2}$. Hence, the rest of the proof is analogous. 